Sādhanā, Vol. 26, Part 4, August 2001, pp. 379-385. C Printed in India

\title{
A note on stability of motion of a projectile
}

\author{
S D NAIK
}

Institute of Armament Technology, Pune 411 025, India

e-mail: insdnaik@yahoo.com

MS received 16 February 2000; revised 12 March 2001

\begin{abstract}
A projectile is stabilised using either gyroscopic or aerodynamic stability. But subcalibre projectiles with sabot have both spin and fins. Separate stability criteria are researched generally for each type of projectile. In this paper a stability criterion which can be used for all such bodies has been developed through the Liapunov second method.
\end{abstract}

Keywords. Projectiles; aerodynamic stability; potential function; spin; sabot; fins.

\section{Introduction}

The basic class of problems in stability of an axis-symmetric body has been discussed by many researchers. Before 1930, the condition $0<\sigma<1$ dominated every design in flight dynamics. Fowler attempted the stability of high angle of fire through his $\beta$-type equations. As awareness of the implications of mathematical modelling to investigate the motion in its full entirety developed during the Second World War, many models were made and linear motion was developed in its completeness. Kebby (1948) investigated nonlinear motion where the parameter is positive and established that if $1-4 q s>0$, the ensuing motion is bounded. Rath \& Sharma (1965) have derived the McShaneMurphy stability condition for slowly yawing motion from Fowler's dynamical equations generalised by Rath taking into account the complete aerodynamic force system (Nielsen \& Synge1946). The motion of a nonlinear Lock-Fowler missile under the same conditon using the Routh-Hurwitz criterion has been discussed by Rath \& Namboodiri (1980).

The dynamical motion of an axi-symmetric projectile in the cross plane moving in the atmosphere (Bakshi \& Sharma 1988) is governed by

$$
\begin{aligned}
& \qquad \ddot{X}+\left(-K_{1} I+K_{2} J\right) \dot{X}+\left(K_{3} I-K_{4} J\right) X=0, \\
& \text { where } J=\left[\begin{array}{cc}
0 & 1 \\
-1 & 0
\end{array}\right], X=\left(\begin{array}{c}
v / u \\
w / u
\end{array}\right), \text { and } \\
& \\
& K_{1}=\varepsilon\left(2 C_{A}-f_{1}\left(g_{2} / K_{t}^{2}\right)\right)-\left(2 g_{x} l / u^{2}\right), \\
& K_{2}=B^{\prime} \varsigma+\varepsilon \varsigma\left(f_{1 p}+\left(g_{2 p} / K_{t}^{2}\right)\right),
\end{aligned}
$$

A list of symbols is given at the end of the paper 


$$
\begin{aligned}
& K_{3}=-\varepsilon\left(B^{\prime} \varsigma^{2} f_{1 p}+\frac{g_{1}}{K_{t}^{2}}\right)+\left(\frac{g_{x} l}{u}\right)^{2}+\frac{g_{x} l}{u^{2}} \frac{\varepsilon}{K_{t}^{2}} g_{2}-\varepsilon\left(\frac{g_{x} l}{u^{2}}\right)\left(2 C_{A}-f_{1}\right), \\
& K_{4}=\varepsilon \varsigma B^{\prime}\left(C_{A}-f_{1}+\frac{g_{1 p}}{K_{t}^{2}}\right)-B^{\prime} \varsigma\left(\frac{g_{x} l}{u^{2}}\right)-\varepsilon \varsigma\left(\frac{g_{x} l}{u^{2}}\right)\left(f_{1 p}+\frac{g_{2 p}}{K_{t}^{2}}\right) .
\end{aligned}
$$

For the spin-stabilized projectile $K_{3}<0$ while for aerodynamically stable projectiles $K_{3}>0$. For a subcalibre projectile with sabot, none of these means can be used simply as a stability criterion, as a spin-stabilised body requires high angular spin and finned stabilised systems need large fins and these fins disturb the sabot design. The better way out is obtained by giving some percentage of spin and smaller fins. Obviously for such a body $K_{3}$ is small with sign indefiniteness. An attempt has been made here to discuss the stability criterion for such a body. It also gives the stability for spin-stabilised as well as fin-stabilised bodies.

\section{Stability criterion}

The P-method (Sharma 1977) has been used here to obtain the generating functions $V$ and $\dot{\mathrm{V}}$.

Let $\quad N \equiv 2 \dot{X}+P X$,

be the generating function. Here $P=P_{1}+P_{2}, P_{1}$ is a symmetric and $P_{2}$ a skew-symmetric $2 \times 2$ matrix. The inner product of (1) and (2) gives

$$
\begin{gathered}
{\left[2 \dot{X}^{t} \dot{X}+\dot{X}^{t} P X+X^{t} P^{t} \dot{X}+X^{t} 2\left(K_{3}+Q\right) \dot{X}\right]} \\
=\dot{X}^{t}\left(P+P^{t}+4 K_{1}\right) \dot{X}+\dot{X}^{t}\left(\left(K_{1} I+K_{2} J\right) P+2 K_{4} J+2 Q\right) \\
X+X+X^{t}\left(\left(K_{1} I+K_{2} J\right) P+2 K_{4} J+2 Q\right)^{t} \\
\dot{X}-X^{t}\left(\left(K_{3} I+K_{4} J\right) P+P^{t}\left(K_{3} I-K_{4} J\right)\right) X,
\end{gathered}
$$

which is of the form $(V)^{\circ}=\dot{V}$. We have introduced an arbitrary matrix $Q$ to develop suitable quadratic forms. The motion is said to be asymptotically stable if

$$
V>0 \text { and } \dot{V}<0 \text {. }
$$

Now $V$ and $\dot{V}$ can be expressed as

$$
V=2\left(\dot{X}^{t}+\frac{X^{t} P^{t}}{2}\right)\left(\dot{X}+\frac{P X}{2}\right)+X^{t}\left(2 K_{3}+2 Q-\frac{P^{t} P}{2}\right) X,
$$

and

$$
\begin{aligned}
& \dot{V}=-\left\{\left[\dot{X}^{t}\left(-\left(P+P^{t}+4 K_{1}\right)\right)^{1 / 2}-X^{t}\left(\left(K_{1} I+K_{2} J\right) P+2 K_{4} J+2 Q\right)^{t}\right.\right. \\
&\left.\times\left(-\left(P+P^{t}+4 K_{1}\right)^{-1}\right)^{1 / 2}\right]\left[\left(-\left(P+P^{t}+4 K_{t}\right)\right)^{1 / 2} \dot{X}-\left(-\left(P+P^{t}+4 K_{1}\right)^{-1}\right)^{1 / 2}\right. \\
& \times\left(\left(K_{1} I+K_{2} J\right) P+\right.\left.\left.2 K_{4} J+2 Q\right) X\right]-X^{t}\left[\left(\left(K_{1} I+K_{2} J\right) P+2 K_{4} J+2 Q\right)^{t}\right. \\
& \times\left(-\left(P+P^{t}+4 K 1\right)\right)^{-1}\left(\left(K_{1} I+K_{2} J\right) P+2 K_{4} J+2 Q\right) \\
&\left.\left.\quad-\left(\left(K_{3} I+K_{4} J\right) P+P^{t}\left(K_{3} I+K_{4} J\right)^{t}\right)\right]\right\} X
\end{aligned}
$$


The asymptotic stability is achieved if $V<0$ and $\dot{V}<0$. That is

$$
2 K_{3}+2 Q-\left(P^{t} P / 2\right) \geq 0
$$

and

$$
\begin{aligned}
\left(K_{3} I+\right. & \left.K_{4} J\right) P+P^{t}\left(K_{3} I+K_{4} J\right)^{t}-\left(\left(K_{1} I+K_{2} J\right) P+2 K_{4} J+2 Q\right)^{t} \\
& \times\left(-\left(P+P^{t}+4 K_{1}\right)\right)^{-1}\left(\left(K_{1} I+K_{2} J\right) P+2 K_{4} J+2 Q\right)>0 .
\end{aligned}
$$

The values of $P$ and $Q$ are arbitrary. One choice of these values (Naik 2000) is

$$
\begin{aligned}
& P=-K_{1} I+K_{2} J, \\
& Q=\left(-K_{3}+\left(P^{t} P / 2\right)\right) I,
\end{aligned}
$$

reduce these conditions to a single condition,

$$
K_{1}^{2} K_{3}+K_{1} K_{2} K_{4}-K_{3}^{2}-K_{4}^{2}>0 .
$$

Another choice for $P$ and $Q$, one can get by generalizing $P$ and $Q$ as

$$
P=2 \frac{K_{2}}{K_{4}}\left(\alpha \frac{K_{2}}{K_{1}} J-\beta I\right),
$$

and $\quad Q=\frac{K_{4} K_{1}}{K_{2}}\left(\beta+\alpha \frac{K_{2}^{2}}{K_{1}^{2}}\right) I$.

These values are selected as $P$ is expressed as $P=p_{1} I+p_{2} J$ and $Q$ is diagonal. $\alpha, \beta$ are scalar quantities. These values of $P$ and $Q$ reduce $V$ and $\dot{V}$ to

$$
\begin{aligned}
V=2\left[\left(\dot{X}^{t}+X^{t} \frac{K_{4}}{K_{2}}\left(-\alpha \frac{K_{2}}{K_{1}} J-\beta I\right)\right)\left(\dot{X}+\frac{K_{4}}{K_{2}}\left(\alpha \frac{K_{2}}{K_{1}} J-\beta I\right)\right)\right. \\
\left.+X^{t}\left(-\frac{K_{4}^{2}}{K_{2}^{2}}\left(\alpha^{2} \frac{K_{2}^{2}}{K_{1}^{2}}+\beta^{2}\right)+K_{3}+\frac{K_{4} K_{1}}{K_{2}}\left(\beta+\alpha \frac{K_{2}^{2}}{K_{1}^{2}}\right)\right) X\right], \quad(1) \\
\dot{V}=4\left(K_{1}-\beta \frac{K_{4}}{K_{2}}\right)\left[\left(\dot{X}^{t}-X^{t}\left(K_{4} J(\alpha-\beta+1) / 2\left(K_{1}-\beta \frac{K_{4}}{K_{2}}\right)\right)\right)\right. \\
\times\left(\dot{X}^{2}\left(K_{4} J(\alpha-\beta+1) / 2\left(K_{1}-\beta \frac{K_{4}}{K_{2}}\right)\right) X\right) \\
-\left(K_{4}^{2}(\alpha-\beta+1)^{2} / 4\left(K_{1}-\beta \frac{K_{4}}{K_{2}}\right)^{2}\right) \\
\left.\left.+\left(\frac{K_{4}}{K_{2}}\left(K_{3} \beta+\alpha \frac{K_{4} K_{2}}{K_{1}}\right) /\left(K_{1}-\beta \frac{K_{4}}{K_{2}}\right)\right)\right],(12)\right]
\end{aligned}
$$

Asymptotic stability is guarranteed for $V>0$ and $\dot{V}<0$ and is obtained if

$$
\begin{aligned}
& -\frac{K_{4}^{2}}{K_{2}^{2}}\left(\alpha^{2} \frac{K_{2}^{2}}{K_{1}^{2}}+\beta^{2}\right)+K_{3}+\frac{K_{4} K_{1}}{K_{2}}\left(\beta+\alpha \frac{K_{2}^{2}}{K_{1}^{2}}\right)>0, \\
& K_{1}-\beta \frac{K_{4}}{K_{2}}<0
\end{aligned}
$$




$$
-K_{4}^{2}(\alpha-\beta+1)^{2}+4 \frac{K_{4}}{K_{2}}\left(K_{1}-\beta \frac{K_{4}}{K_{2}}\right)\left(K_{3} \beta+\alpha \frac{K_{4} K_{2}}{K_{1}}\right)>0 .
$$

$\alpha$ and $\beta$ can be arbitrary. In particular, $\alpha=1, \beta=0$ reduce (13) to

$$
\begin{aligned}
& K_{1}<0 \\
& -\left(K_{4}^{2} / K_{1}^{2}\right)+K_{3}+\left(K_{4} K_{2} / K_{1}\right)>0, \\
& K_{1}^{2} K_{3}+K_{1} K_{2} K_{4}-K_{4}^{2}>0,
\end{aligned}
$$

which are conditions for spin-stability. Similarly $\alpha=0, \beta=1$, reduce the above conditions (13) to

$$
\begin{aligned}
& K_{4} / K_{2}<0, \\
& K_{1}-\left(K_{4} / K_{2}\right)<0, \\
& K_{3}>0 .
\end{aligned}
$$

These are the conditions for fin-stability. For a body which is stabilised with small spin and fins, one can select $\alpha$ and $\beta$ that are arbitrary but real and nonzero. A linear relation between $\alpha$ and $\beta$ has been attempted here. On examining various values, the one that is applicable is

$$
\alpha-\beta=1 \text {. }
$$

This reduces the stability conditions to

$$
\begin{aligned}
& -\frac{K_{4}^{2}}{K_{2}^{2}}\left((1-\beta)^{2} \frac{K_{2}^{2}}{K_{1}^{2}}+\beta^{2}\right)+K_{3}+\frac{K_{4} K_{1}}{K_{2}}\left(\beta+(1-\beta) \frac{K_{2}^{2}}{K_{1}^{2}}\right)>0, \\
& K_{1}-\beta\left(K_{4} / K_{2}\right)<0, \\
& -K_{4}^{2}\left(1-\beta^{2}\right)+\frac{K_{4}}{K_{2}}\left(K_{1}-\frac{K_{4}}{K_{2}} \beta\right)\left(K_{3} \beta+(1-\beta) \frac{K_{4} K_{2}}{K_{1}}\right)>0 .
\end{aligned}
$$

The value of $\beta$ can be selected in such a way that $V>0$. Let

$$
\begin{aligned}
& \Psi(\beta)=- \frac{K_{4}^{2}\left(K_{1}^{2}+K_{2}^{2}\right)}{K_{1}^{2} K_{2}^{2}} \beta^{2}+\frac{K_{4}}{K_{1} K_{2}}\left(K_{1}^{2}+K_{2}^{2}-\frac{2 K_{4} K_{2}}{K_{1}}\right) \beta \\
&+\frac{1}{K_{1}^{2}}\left(-K_{4}^{2}+K_{3} K_{1}^{2}+K_{1} K_{2} K_{4}\right) .
\end{aligned}
$$

The condition for $V>0$ is $\Psi(\beta)>0 . \Psi(\beta)$ is a quadratic in $\beta$ and has two roots. Its maximum value should provide a practical design situation and is obtained when the two roots are equal.

$$
\begin{aligned}
& \beta_{\max }=\left(K_{1} K_{2} / 2 K_{4}\right)\left(1-\left[2 K_{2} K_{4} / K_{1}\left(K_{1}^{2}+K_{2}^{2}\right)\right]\right), \\
& \Psi(\beta)>0, \text { implies } \\
& K_{1}^{2}+K_{2}^{2}+4 K_{3}-\left[4 K_{4}^{2} /\left(K_{1}^{2}+K_{2}^{2}\right)\right]>0 .
\end{aligned}
$$


It is same as (16(a)). The last term in the above equation appears through the interaction of spin and fins combination stability. This term being positive decreases the spin stability situation. This arises due to the coupling in magnus and damping coefficients. We need a strongly damping force due to spin appearing in $K_{1}$.

For a subcalibre projectile which is stabilized with spin as well as fins, condition (18) can be called the dynamic stability condition. The corresponding condition for spinning bodies is $K_{1}^{2}+K_{2}^{2}+4 K_{3} \geq 0$. If $K_{2}^{2}+4 K_{3} \geq 0$, the above condition is always satisfied. This is static stability for a spinning body.

In particular, if we ignore damping parameter $K_{1}$, the dynamic stability (18) becomes $K_{2}^{2}+4 K_{3}-\left(4 K_{4}^{2} / K_{2}^{2}\right) \geq 0$.

This contains a parameter $\mu=K_{4} / K_{2}$ which is very important for the design. For this, $\beta_{\max }=-1$, gives the fin stability.

With this $\beta_{\max }$ defined as (17), the other two conditions for asymptotic stability coming from $\dot{V}<0$ are

$$
\begin{aligned}
& \left(K_{1} / 2\right)+\left(K_{2} K_{4} /\left(K_{1}^{2}+K_{2}^{2}\right)\right)<0, \\
& \left(K_{1}^{2}+K_{2}^{2}\right)^{2}\left(K_{1}^{2} K_{3}+K_{1} K_{2} K_{4}-2 K_{4}^{2}\right)+4 K_{2} K_{4}^{2}\left(K_{1} K_{4}-K_{2} K_{3}\right) \geq 0 .
\end{aligned}
$$

The condition (20) for a marginally stable projectile, $K_{3}=0$, and a damped system $K_{1}<0$ implies that $K_{4}$, the magnus coefficient should be negative, a situation which is a generally a design situation. Hence this result is likely to be met. This negative magnus coefficient also strongly satisfies (19).

Thus a negative magnus coefficient with a marginal value as sign indefinite is necessary for stability of a subcalibre projectile.

\section{Verification}

Let us consider a projectile with the following data.

$$
\begin{aligned}
\rho & =1.125, \\
m & =3.6, \\
d & =0.028, \\
p & =145, \\
u & =1447, \\
l & =0.486, \\
C_{D} & =0.35, \\
f_{1} & =12.9, \\
g_{1} & =2.5, \\
g_{2} & =-574.6, \\
g_{2 p} & =-25.86, \\
I_{x x} & =3.66, \\
I_{y y} & =549.06 .
\end{aligned}
$$

To verify the stability criteria, we have to calculate the parameters $K_{1}, K_{2}, K_{3}$ and $K_{4}$ first. Here

$$
\varepsilon=\rho S d / 2 m, \quad K_{t}^{2}=I_{y y} / m d, \quad B^{\prime}=I_{x x} / I_{y y} \quad \text { and } \quad \varsigma=p l / u .
$$

Generally $\varepsilon^{2}$ and $\Omega_{x}^{-}$are small and ignored. The values of $K_{i}$ 's are simplified to $K_{1}=-3.742 \times 10^{-5}$ 
$K_{2}=0.00203796$
$K_{3}=0.454 \times 10^{-10}$
$K_{4}=-0.007354 \times 10^{-5}$.

It can be further observed that

(a) All the conditions (8), (18),(19) and (20) are satisfied.

(b) The spin required for stability of this projectile is $140 \mathrm{rev} / \mathrm{s}$. Hence the projectile is over-stabilized as actually the spin applied is $145 \mathrm{rev} / \mathrm{s}$. It is thus concluded that sufficient stability criterion meets the technology requirements.

(c) To stabilize this projectile only with the help of spin ,the requirement is $387 \mathrm{rev} / \mathrm{s}$. The spin can be reduced by introducing small fins in the design. In this design the energy saved because of the fins is $85 \%$ of that of the spin-stabilised projectile, which can be utilised in any other form.

\section{Conclusions}

A simple analysis has provided very important design critera for a subcalibre projectile. These are as below.

(1) The spin-stability is determined by fins through the magnus parameter. This will be smaller if fins are smaller which is the case for subcalibre projectile.

(2) The magnus moment has to be negative.

(3) The negativeness of the magnus moment damps the oscillations faster as seen from (19).

\section{List of symbols}

$C_{D}$ drag force coefficient;

$\mathrm{d}$ diameter of the projectile;

$f_{1} \quad$ normal force coefficient;

$g_{1} \quad$ aerodynamic moment coefficient;

$g_{2}$ damping moment coefficient;

$g_{2 p}$ magnus moment coefficient;

$l$ total length of the projectile;

$I_{x x}$ moment of inertia of the projectile about $X$-axis;

$I_{y y}$ moment of inertia of the projectile about $Y$-axis;

$K_{1} \quad$ damping parameter;

$K_{2} \quad$ spin parameter;

$K_{3}$ conservative force parameter;

$K_{4}$ magnus parameter;

$\mathrm{m}$ mass of the projectile ;

$p \quad$ constant spin of projectile;

$\rho$ density of air;

$u \quad$ avarage speed of the projectile.

\section{References}

Bakshi S, Sharma K C 1988 A fresh look at the free flight dynamics of spinning projectile. Sadhana 12: 321-337 
Kebby C H 1948 The stability of the undamped motion of a projectile subject to a yawing moment of the Fowler-Lock type. Armament Research Establishment Report No. 38/48 Naik S D 2000 Stability criterion for a finned spinning projectile. Int. J. Differential Eqnations Dyn. Syst. 8: 151-163

Namboodiri A V 1980 On the dynamic response of a rolling missile due to a nonlinear aerodynamic moment of the Lock-Fowler type. Ph D thesis, Department of Mathematics, University of Poona, Pune

Nielsen K L, Synge J L 1946 On the motion of a spinning shell. Q. J. Appl. Math. 4: 201-206

Rath P C, Sharma J P 1965 On the stability of a spinning projectile. Des. Sci. J. 15: 237-250

Sharma K C 1977 On the dynamic stability of a missile. Des. Sci. J. 27: 29-32 